\title{
Adolescent to Adult Patient-centered HIV Transition \\ (ADAPT): Study Protocol for a Cluster Randomized Trial
}

\section{Ernest Ekong ( $\nabla$ ernestekong@ihvnigeria.org ) \\ Institute of Human Virology https://orcid.org/0000-0003-0385-1882}

\section{Chidozie Edokwe}

Institute of Human Virology

Christopher Isah

Institute of Human Virology

\section{Ashley Shutt}

University of Maryland

\section{Manhattan Charurat}

University of Maryland

\section{Fatimah Hassan-Hanga}

Aminu Kano Teaching Hospital

Ismail Buhari

General Hospital Katsina

\section{Mfon Essen}

Federal Medical Center Keffi

\section{Esther Audu}

Path Dalhatu Araf Specialist Hospital

\section{Adaora Okechukwu}

University of Abuja Teaching Hospital

Henry Onyegbutulem

Asokoro District Hospital

\section{Vicki Tepper}

University of Maryland

\section{Method Article}

Keywords: HIV, Healthcare transition, Adolescent, Retention, Viral suppression, Nigeria

Posted Date: January 4th, 2019

DOI: https://doi.org/10.21203/rs.2.175/v1

License: (c) (i) This work is licensed under a Creative Commons Attribution 4.0 International License. Read Full License 


\section{Abstract}

Background: Innovative strategies and protocols are required for effective provision of healthcare services and attainment of optimal positive health outcomes for adolescents living with HIV (ALHIV) as they transition to adult care.HIV-infected youth must learn to navigate a complex healthcare system as they transition from pediatric to adult care. Poorly planned transitions can result in non-adherence to ART, loss-to-follow-up in care and supportive services, with resultant negative health impact. This study evaluates two different approaches to the process of transition from pediatric to adult care in six facilities in Nigeria.

Methods: ADAPT will use a cluster randomized intervention trial to measure the impact of Peer Transition Advocates (PTAs) versus Education Interventionists (Els) approach to the process of transition of ALHIV, with imbedded psychosocial/social network analyses. The study will be conducted in 6 healthcare facilities (three tertiary and three secondary) in Northern and Central Nigeria. The unit of randomization (cluster) will be the facilities ( $n=180$ per intervention arm, $\mathrm{N}=360$ ), study participants 17-19 year-old ALHIV enrolled in the pediatric ART clinic for $\geq 12$ months and know their HIV status. Sixty participants will be recruited pre-transition per site and followed up 12 months posttransition. Primary outcome measure will be proportion of ALHIV achieving successful transition and viral suppression 12 months post-transition comparing the two arms. Secondary outcome measures are proportion of ALHIV achieving ownership of care, improved quality of life, better transfer experience, and social support system measured by individual and ego-network evaluation tools correlating with successful transition.

Discussion: ADAPT hypothesizes that by involving ALHIV in the study design, the PTA intervention will result in more successful transition and clinical outcomes in adult care. Further, we postulate that properly trained and supervised task-oriented peer support using lay workers assigned to track and guide each adolescent along the transition pathway in the clinic and in the community is the key starting point for transforming a dysfunctional system. Findings from ADAPT will guide institutions on best practices for transitioning ALHIV and other adolescents with chronic conditions in Nigeria and other resource-limited settings.

\section{Background}

The number of adolescents and young people living with HIV continues to increase globally $(1,2)$. According to UNICEF (1), 610,000 young people aged 15 to 24 years, including 260,000 adolescents between 15 and 19 years of were newly infected with HIV in 2016. Nigerian national data indicates that $4.2 \%$ of young people (ages 15-24 years) are living with HIV, including an estimated 160,000 adolescents aged 10-19 (3). Nigerian adolescents represent $17 \%$ worldwide burden of all older ALHIV [15-19 year-olds]. Furthermore, this number is expected to increase with improved survival among perinatally infected children and new behaviorally acquired infections, which make up $30 \%$ of new HIV infection in Nigeria. Idele et al. (4) reported that among the five countries with the highest burden of HIV infection among adolescents - South Africa, Nigeria, Kenya, Tanzania, and Uganda - only Nigeria is still experiencing an increase in young adolescent deaths (4). Early sexual debut is common in Nigeria, often beginning at less than 15 years for $15 \%$ of Nigeria's youth. This increases HIV vulnerability among young people. In addition, Nigeria has reported very low uptake of HIV testing, resulting in only $17 \%$ of HIV infected young people knowing their status (5).

One of the challenges facing adolescents living with HIV disease is the transition of their care in a pediatric program to the adult health care setting. Although studies $(6,7,8,9)$ have explored linkages from diagnosis to care and treatment of newly diagnosed HIV adolescents, little work has been done to examine related barriers to engagement in care within the adult health care context. Newly diagnosed adolescents struggle with HIV-related stigma (from self and others) and 'shame' (10) and often have a poor understanding of their disease. In addition, structural barriers such 
as lack of transportation may impact access and engagement in care. Transition-related barriers include a lack of inter-clinic communication (11), the unwillingness of the adolescents and their caregivers to transition from the pediatric care setting, adolescent providers' unwillingness to relinquish care (12) and adult caregivers' limited adolescent-specific training (10). Adult clinics, unlike adolescent clinics, are less flexible with missed appointments and provide minimal case management (13) and are HIV-specific and not comprehensive enough for the needs of emerging adults $(14,15)$.

The transition from pediatric to adult medical care involves more than just changing the location of services from the pediatric to the adult clinics (16). ALHIV are expected to assume responsibility for their own health and disease management (17). This is a major challenge for sustained retention and adherence along the care continuum.

\section{Study objectives and Hypotheses}

The objective of ADAPT is to answer critical questions of how to best support and sustain successful transition for ALHIV and whether a locally designed and patient-centered approach improves engagement of ALHIV in transition resulting in improved clinical outcomes.

The specific aims of are:

- To measure the evaluate the efficacy of a peer supported approach to transition compared with an educational approach to the process of transition

- To identify demographic, social support network, psychosocial correlates of successful transition and clinical outcomes.

We hypothesize that:

\section{A patient-centered approach will improve the process of transition}

2. A transition intervention that is co-designed with ALHIV will result in improved transition and clinical outcomes

3. Social support network and psychosocial factors are essential for successful transition.

To guide the study design and the development of the interventions, qualitative focus groups (FGD) were conducted to obtain input and direction from ALHIV, parents and health care providers. The focus groups were comprised of ALHIV who had not begun the transition process, who successfully transitioned, and who had unsuccessful transitions as defined by their health care team. Other groups were comprised of parents of ALHIV and health care providers from the 6 study sites. Participants were interviewed by a researcher using a script containing a series of open-ended questions in key domains: the experience of having the health care provider introduce the concept of transition, ALHIV readiness for and importance of transition, advantages and disadvantages of transition, and what constitutes a successful transition. In addition, the acceptability of education and advocate approaches, and personnel and resources to support the transition process were reviewed with all focus group participants. The focus group participants provided feedback on the cultural relevance and appropriateness of the measures to be used. Further, input on the implementation plan for recruitment and retention of participants in the study was also obtained. Based on this protocol, informed consent and assent, as well as data collection instruments were developed.

\section{Methods}

\section{Study Design}


ADAPT is a cluster randomized trial (RCT) of the effect of either a peer transition advocate (PTA) or transition educational interventionist (EI) on adolescent outcomes following transition from pediatric to adult medical care. (Figure 1).

\section{Phase II Description of Intervention Arms}

\section{Peer Transition Advocate Approach.}

The transition advocate arm of the intervention will be delivered by three peer transition advocates (PTAs), one based at each site. Selection and training: Candidate PTAs will be nominated by facility staff and selected in consultation with implementing partners (IP) and research study staff. PTAs may be drawn from current peer support groups and must be living with HIV or - affected with personal experience with HIV. Criteria include resident in or readily accessible to the area served by the facility, ability to read and write simple English, ability to establish rapport, fluency in local language, and willingness to disclose their own status. Formal training will be conducted to cover the basics of HIV and transition process, program goals and structure, transmission risks, treatment, adherence, and schedule of services. Skills in communications and counseling, effective ways to address stigma and disclosure, confidentiality, documentation, and linkage to the community and clinical resources will be taught the candidates. Training will emphasize interactive activities such as role-playing. Duties: The PTAs will provide individualized coaching, modeling, support and linkage of participants and their families to adult HIV care providers in the same facility, and follow adolescent patients until after 12-months post-transition. The PTAs will be present during patient clinic appointments to mentor and support participants in the development of independent healthcare behaviors (e.g., making follow up appointments). Their duties will be performed in the clinic and in the community and will span from identification and referral linkage from HIV testing and counseling to tracking and defaulter tracing of patients. Each encounter-in the clinic or by phone- will be documented in a log. PTAs will be supervised by the NC and, when there are clinical concerns, to a designated clinician at the adult clinic. PTAs will also reach out to patients aged 16 years, to engage and prepare them for the transition process. The specific approach and format of the support that the PTAs will offer will be refined with feedback from our focus groups in the first year of the project so that we are addressing the needs that patients identify as most desired and needed during the transition process. PTAs caseload will approximate 10 new ALHIV/year or about 25 patients at steady state.

\section{Education Interventionist Approach.}

An educational approach for supporting the transition from pediatric to adult HIV care is being compared against the PTA approach described above. The education intervention arm of the trial will be implemented by three education interventionists (Els), selected and hired by the referral facilities. Prior to the initiation of the intervention, an individualized transition plan will be developed to address patient needs identified in the assessment of readiness for transition. The educational intervention will include the use of handouts, other educational materials and pertinent information needed for the transition process. The El will be present to explain information to participants and their parents but they will not accompany participants to appointments in the adult setting.

\section{Study setting}

The study will be conducted within the setting of the President's Emergency Plan for AIDS Relief (PEPFAR) program in Nigeria operated by the Institute of Human Virology Nigeria (IHVN) in four states - the Federal Capital Territory (FCT), Nassarawa, Kano, and Katsina. The study sites are geographically located in central and northern Nigeria. They typify characteristics of Nigeria: moderate-low HIV prevalence compared to Southern and Eastern African nations but highest in Nigeria. They have varied ratios of Christians to Muslims ( $50 \%$ Muslims in north-central states and $<5 \%$ in 
south-central states), and various ethnic groups (18). IHVN supports more than 395 facilities in these states with HIV prevention, treatment and care services to people living with HIV including 10-19 years old ALHIV.

\section{Study site selection and randomization}

The study will be conducted in six secondary and tertiary healthcare facilities. These facilities include Aminu Kano Teaching Hospital, Kano; University of Abuja Teaching Hospital, Abuja, FCT; Dalhatu-Araf Specialist Hospital, Lafia, Nassarawa State (tertiary facilities); Asokoro District Hospital, Abuja; Federal Medical Centre, Keffi, Nassarawa State; and General Hospital Katsina, Katsina (secondary facilities). A total of 479 adolescents are currently receiving HIV care at these 6 sites as of October 11,2016, during the period of the site selection. The process of site selection involved several months of site visits and abstraction of disaggregated PEPFAR indicator data. In addition, two facilities were identified as reserved sites. The study sites were selected based on:

- Having at least 60 adolescents enrolled and retained in care for at least 2 years

- Having at least one dedicated pediatric physician, one adult physician and one nurse in the ART clinic

- Accessibility of the research team to the sites

- Willingness of the sites to collaborate

Table 1: Characteristics of study sites

\begin{tabular}{|c|c|c|c|c|c|c|}
\hline $\mathrm{S} / \mathrm{N}$ & Name of Facility & $\begin{array}{l}\text { Facility } \\
\text { type }\end{array}$ & $\begin{array}{l}\text { No. of adolescents } \\
\text { enrolled (2014- } \\
\text { 2015) }\end{array}$ & $\begin{array}{l}\text { No. of adolescents } \\
\text { retained in care (2014- } \\
2015 \text { ) }\end{array}$ & State & Status \\
\hline 1. & $\begin{array}{l}\text { University of Abuja } \\
\text { Teaching Hospital } \\
\text { (UATH) }\end{array}$ & Tertiary & 74 & 64 & FCT & Selected \\
\hline 2. & $\begin{array}{l}\text { Asokoro District } \\
\text { Hospital }\end{array}$ & Secondary & 67 & 60 & FCT & Selected \\
\hline 3. & $\begin{array}{l}\text { Aminu Kano Teaching } \\
\text { Hospital (AKTH) }\end{array}$ & Tertiary & 69 & 56 & Kano & Selected \\
\hline 4. & $\begin{array}{l}\text { General Hospital, } \\
\text { Katsina }\end{array}$ & Secondary & 41 & 29 & Katsina & Selected \\
\hline 5. & $\begin{array}{l}\text { Federal Medical Centre, } \\
\text { Keffi }\end{array}$ & Tertiary & 99 & 76 & Nassarawa & Selected \\
\hline 6. & $\begin{array}{l}\text { Dalhatu Araf Specialist } \\
\text { Hospital, Lafia }\end{array}$ & Secondary & 103 & 94 & Nassarawa & Selected \\
\hline 7. & Sev-Av Vandeikya & Secondary & 48 & 45 & Benue & Reserved \\
\hline 8. & $\begin{array}{l}\text { Mararaba Guruku } \\
\text { Medical Center }\end{array}$ & Secondary & 48 & 37 & Nassarawa & Reserved \\
\hline
\end{tabular}

The unit of randomization (cluster) will be the facilities. The six facilities will be randomized into two separate intervention arms (PTA vs EI). The randomization process will be computer based.

\section{Study population}

Study participants will be selected from among ALHIV enrolled in and receiving care at participating healthcare facilities.

Inclusion and exclusion criteria 
Inclusion Criteria:

ALHIV who meet the following criteria will be eligible for enrollment in ADAPT:

1. Two documented positive HIV-1 rapid serologic tests in accordance with the Nigerian National Testing algorithm. They are eligible regardless of mode of transmission, and regardless of whether the mode of transmission is known.

2. AHLIV 17-19 years of age.

3. Enrollment in HIV care for $\geq 12$ months at study site and has been treated with ART.

4. The patient is aware of their HIV status.

Exclusion Criteria:

1. Patient has documented significant cognitive impairment

2. The patient is planning to transition HIV care outside the selected study facilities

3. Patient or caregiver (for minors) fails to provide informed consent/assent

\section{Participant recruitment}

Eligible patients will be identified by clinic staff at which time clinic staff will inquire about their interest in learning about the study. If interested in study participation, the Nurse Coordinator will explain the nature, purpose, and requirements of the study to the patient and the parent/guardian. Questions about the study will be solicited, and informed consent procedures will be conducted in the primary language of the patient (and caregiver if the patient is a minor). The parent/guardian will provide informed consent, while the patient will assent to participate in the study. Participants who are 18 - 19 years of age will provide their own informed consent. Following consent/assent, baseline data collection will be initiated. Patient participation will also be solicited through the use of a flyer advertising the study that includes the contact information of study staff at the clinic sites. For those who are recruited in this manner, study eligibility will be confirmed by the patient and a core clinical staff member and informed consent procedures will be conducted as described above.

\section{Enrollment Process:}

Participants will complete the baseline surveys/assessments during regularly scheduled clinic visits. Each participant will receive 1,000 Naira as reimbursement for transportation. Following baseline data collection, the participant will be contacted by either the PTA or El (based on site randomization) to be notified of the day and time of their next appointment. Participants will receive phone calls and/or text messages to maintain engagement with the study staff. The window for the completion of these activities will be from one week prior, to 21 days after the identified baseline data collection date.

\section{Sample size estimation}

With a mean of 60 ALHIV per referral facility, there will be a minimum of 180 participants per intervention arm. Available findings on HIV counseling and testing suggest a $50 \%$ rate of successful transition outcomes among ALHIV with data on the advocacy-based approach (19) and the education-based approach showing a successful transition to be $80 \%$ and $40 \%$, respectively. Based on these estimates, the study is more than sufficient to provide $>80 \%$ power to 
detect a $30 \%$ effect size difference in proportion retained at 12 months post-transition between two groups at the intracluster correlation (ICC) of 0.05 and a significance level of 0.05 .

\section{Data collection}

Socio-demographic and medical history information will be collected from the study participants and/or caregivers via face-to-face interview. Other source documents will include patients' case notes, clinical notes, clinical charts, laboratory result and standardized study forms (described below). We expect this to take from $30-60$ minutes. The standardized study forms will be managed by the research team and data manager on the study team. Clinicians, other health personnel working in the hospital and members of the study team will have access to these records. The clinical site will maintain appropriate medical and research records for this study, in compliance with The International Council for Harmonization of Technical Requirements for Pharmaceuticals for Human Use (ICH) E6, Section 4.9 and regulatory and institutional requirements for the protection of the confidentiality of subjects (20). Standardized study forms will also be completed at transition, 6 months and 12 months post-transition respectively (Table 2).

\section{Table 2: Schedule of data collection}

\begin{tabular}{|l|l|l|l|l|}
\hline Activity & Baseline & At transition (T) & T + 6months & T + 12months \\
& & & & \\
\hline Patient demographic data & $\mathrm{X}$ & & & \\
\hline Patient clinical data & $\mathrm{X}$ & & $\mathrm{X}$ & $\mathrm{X}$ \\
\hline CD4 count & $\mathrm{X}$ & & $\mathrm{X}$ & $\mathrm{X}$ \\
\hline Plasma viral load & $\mathrm{X}$ & $\mathrm{X}$ & $\mathrm{X}$ & $\mathrm{X}$ \\
\hline Plasma storage & $\mathrm{X}$ & & $\mathrm{X}$ & $\mathrm{X}$ \\
\hline Ease of transition & & $\mathrm{X}$ & & \\
\hline Communication with providers & $\mathrm{X}$ & & $\mathrm{X}$ & $\mathrm{X}$ \\
\hline Patient-centered measures of disease control & $\mathrm{X}$ & & $\mathrm{X}$ & \\
\hline Patient Activation Measure (PAM) & $\mathrm{X}$ & $\mathrm{X}$ & $\mathrm{X}$ & $\mathrm{X}$ \\
\hline Pediatric Quality of Life Inventory (PedsQL) & $\mathrm{X}$ & & $\mathrm{X}$ & $\mathrm{X}$ \\
\hline Network data measured in ego-alter format & $\mathrm{X}$ & & $\mathrm{X}$ & $\mathrm{X}$ \\
\hline Successful Transition & & & & $\mathrm{X}$ \\
\hline
\end{tabular}

Following the same plan, plasma viral load (pVL) and CD4 counts will be measured and the results will be transmitted and filed in participants' medical record for use by the patients' clinician. The Nigerian national treatment guidelines stipulates that stable patients on ART for at least 6 months should have at least one viral load test every year (21). Each clinic visit will be documented to track and measure primary outcome of retention in care.

Table 3: Description of evaluation tools 


\begin{tabular}{|c|c|c|c|c|}
\hline Measures & Tools & How to measure & Validation \& prior use & $\begin{array}{l}\text { Time to } \\
\text { complete }\end{array}$ \\
\hline $\begin{array}{l}\text { Inclusion and } \\
\text { respect in } \\
\text { health care } \\
\text { decisions }\end{array}$ & $\begin{array}{l}\text { Patient Activation } \\
\text { Measure (PAM) }\end{array}$ & $\begin{array}{l}\text { Perceptions and ownership of } \\
\text { care with } 4 \text { activation levels along } \\
\text { an empirically derived continuum. }\end{array}$ & $\begin{array}{l}\text { 13-items, Cronbach's a = } \\
0.87 . \text { Validated in multiple } \\
\text { diseases including HIV } \\
\text { (27) }\end{array}$ & 10 mins \\
\hline $\begin{array}{l}\text { Good quality } \\
\text { of life }\end{array}$ & $\begin{array}{l}\text { Pediatric Quality of } \\
\text { Life Inventory } \\
\text { (PedsQL) }\end{array}$ & $\begin{array}{l}\text { Health-related quality of life for } \\
\text { adolescents with chronic health } \\
\text { conditions. }\end{array}$ & $\begin{array}{l}\text { 15-items, Cronbach's a = } \\
0.90 . \text { Validated in multiple } \\
\text { diseases including HIV } \\
(28)\end{array}$ & 4 mins \\
\hline $\begin{array}{l}\text { Perception of } \\
\text { care and } \\
\text { services }\end{array}$ & $\begin{array}{l}\text { Patient } \\
\text { Satisfaction } \\
\text { Survey }\end{array}$ & $\begin{array}{l}\text { Evaluates perception and } \\
\text { satisfaction of care \& services } \\
\text { received from healthcare facilities }\end{array}$ & 24-items & 8 mins \\
\hline Social Capital & $\begin{array}{l}\text { Social Support and } \\
\text { Network Survey }\end{array}$ & $\begin{array}{l}\text { Composition, influence and } \\
\text { network of adolescent social life }\end{array}$ & 23-items & 8 mins \\
\hline $\begin{array}{l}\text { Transition } \\
\text { Readiness }\end{array}$ & $\begin{array}{l}\text { Transition } \\
\text { Readiness } \\
\text { Assessment } \\
\text { Questionnaire } \\
\text { (TRAQ) }\end{array}$ & $\begin{array}{l}\text { Assess skill level in areas } \\
\text { important for transition to adult } \\
\text { care }\end{array}$ & 18-item (43) & 5 mins \\
\hline
\end{tabular}

\section{Ownership of care, satisfaction.}

Assessment of patient's readiness for engagement in health care and quality of life will be measured. All measures have been presented to participants in the focus groups and were modified so that the language and questions were relevant and appropriate in the Nigerian context. The Patient Activation Measure (PAM) assessment gauges the knowledge, skills, and confidence essential to managing one's own health and healthcare. The 13-item survey has been well psychometrically tested and validated in many settings including HIV management (22). It is unidimensional in that it taps into an overarching construct: being in charge of one's own health. The Pediatric Quality of Life Inventory (PedsQL) is a 15-item tool designed to measure health-related quality of life in children and adolescents using core dimensions of health delineated by the World Health Organization (23). The core scales measure physical functioning (8-items), emotional functioning (5-items), social functioning (5-items), and school functioning (5-items). It is responsive to clinical change over time and has been translated into multiple languages.

\section{Social support network.}

Network data will be compiled based on information collected in the ego-alter format. Respondents (egos) in personal network studies are usually asked to report information about members of their personal network (alters) (24). Egos respond based on their personal perceptions. In this study, egos will be asked to name alters who would provide different types of social support. Also, the network relationship between ego and corresponding alters will be categorized into friends, family members, and others identified by the ego. The closeness of the relationship, size of social network, and frequency of ego-alter contacts will be categorized accordingly. Network social support determined by the proportion of the total available network members to provide support will be determined. This framework will then be used to identify differences that may exist between ALHIV with the successful transition and those whose transition was not successful. This will improve our ability to refine effective interventions to increase the successful transition that are tailored to the needs of each group. These measurements will be performed according to the schedule in Table 2 by nurse coordinators who speak the primary local language.

\section{Specimen collection}

Blood samples of study participants will be drawn for viral load testing via venipuncture. The samples will be collected at enrollment, at transition, 6 months and 12 months post transition (Table 2). To ensure confidentiality, 
participants' names will never be recorded on any specimens, specimen manifest, or inventory sheets designed for study use. Only de-identified study ID will be used.

To ensure quality and uniformity, all processed samples from sites will be transported and tested at a central laboratory. A standard operating procedure (SOP) and logistics for transportation of samples will be carefully designed.

\section{Outcome analyses}

The study focuses on a service primary outcome, successful transition, as evidenced from two consecutively kept appointments at the designated adult clinic within 9-12 months of transition, and a clinical primary outcome, $p V L<400$ at 12 months post-transition. Primary analyses will be on an intention-to-treat (ITT), missing equals failure basis. To assess the effect of the intervention on successful transitioning and viral suppression, multivariate logistic regression models will be applied.

Virologic failure would be likely to have occurred in participants LTFU, with consequent risks of disease progression. A major focus of ADAPT is to study systems, and successful transition is a cardinal measure of systems function. We will also focus on clinical outcomes, Secondary analyses will explore alternatives with sensitivity analysis and include different approaches and also apply objective measures of patient's ownership in health, quality of life, and satisfaction. The relationship between the randomized interventions and primary and secondary process and clinical outcomes will be analyzed, with the opportunity to observe where failure to complete interventions occurs along the path of the transition process. These observations will be correlated with demographic characteristics, attitudes towards clinical care, and psychosocial. Psychosocial functioning will be tracked by longitudinal scores from tools described in Table 3.

\section{Primary outcome analysis.}

Successful transition will be defined as being retained in care post-transition with at least 2 clinical visits within a 12month period (in Nigeria, patients are given quarterly clinical appointments). The proportion of patients achieving this primary outcome will be compared using chi-square test. We will calculate cluster-specific outcomes for each cluster and compare the differences between health facilities with PTA and those with El. To assess the effect of the intervention (PTA vs. EI) on successful transitioning, multivariate logistic regression models with generalized estimating equation (GEE) will be used to account for clusters. First, univariate models for associations between successful transition, type of intervention, and individual characteristics will be developed. To minimize bias, biologically plausible confounders and variables that will change crude estimate by $5 \%$ will be sequentially added to the multivariate model. This will be stopped when addition will not result in a further change in estimate. We will also estimate ICC from these models. We anticipate a low non-participation rate based on our previous studies. Characteristics of patients who decline will be compared with participants to look for potential bias, including the number of individuals who presented to clinics and refused consent or were lost to follow up prior to being offered study participation. $\mathrm{pVL}<400$ at 12 months post-transition: the proportion of patients achieving this primary outcome will be compared between arms by chi-square test and logistic regression adjusting for clustering $(25,26)$ and baseline characteristics (if necessary). For primary outcomes, we will present our results according to the CONSORT guideline (27) for reporting clinical trials. Interim analysis of primary outcomes will be conducted at 2 years into the project to see if one intervention is more effective than another.

\section{Transition pathway analysis}


To determine whether the intervention effect and different risk factors are associated with points in the transition pathway, we will employ a landmark analysis $(28,29)$ to define factors associated with risk of not completing each step in the pathway defined in Figure 4 using each step completion as the outcome. This approach allows us to characterize risk factors that may be unique at each step or continuously associated with multiple steps.

In addition to estimating an average treatment effect, the study will examine intervention effects in clinically relevant subgroups by stratifying the primary analyses by HIV disease stage at transition (as measured by CD4+ T-lymphocyte, $\mathrm{pVL}$ ), gender and presence of co-morbidities. Earliest pVL collected at 3 months post-transition will also be evaluated. The limited information that is available suggests that male adolescents may have poorer experience and lower successful transition than female adolescents (30), but there is a paucity of data in the resource-limited setting. In the multivariate analyses, we will account for the underlying variability in the data by using interaction terms.

\section{Quality of life and ownership in health analysis}

We will calculate mean changes in Patient Activation Measure (PAM) and quality of life from baseline to 6-month and 12-month post-transition visits between groups using t-test and analysis of variance test. We will evaluate whether PAM collected at the time of transition predicts subsequent PAM at 6- and 12-month post transition. For quality of life, we will introduce the PedsQL in the primary outcome analysis models to further examine whether patient's ownership in health and quality of life affect patient's defined outcomes and successful transition.

\section{Social network analysis}

To examine the potential role of social network components and characteristics of both egos and alters on primary outcomes, GEE will be used to explore the associations between primary outcomes and factors at the ego, alter, and network levels. As observations within an egocentric network are highly dependent, traditional regression models that assume independent observations cannot be used. GEE models adjust for the dependent observations within an ego's network. The independent variables will be social network factors (i.e. relationship, trust in alters, contact frequency, social support, and network size) as well as social demographic variables of both egos and alters. To investigate their interrelationships, a hierarchical combination of candidate variables will be considered for model building. Because there is no likelihood estimation for GEE models, quasi-likelihood information criterion (QIC) and QICu will be used as the model selection criteria. The smaller the values of the QIC and QICu, the better the model will be to describe the relationship.

\section{Data management}

The Study Database System for all study forms is integrated with the Study Visit Management System. During data capture, all fields are checked with range and logic checks. In addition to the data forms being reviewed on-site, some of the forms will also be reviewed by the central research team. Each participant will complete a 6 month and 12 months follow up visit. For reasons of virologic failure, a 3 month follow up visit will be required for such patient. This follow-up will include collecting data and updating any information used to contact the participant. To minimize risk, all records will be kept confidential, all data will be secured electronically by storing it in a computer that is password protected and not connected to the internet. Any research data that is shared among study staff will be de-identified and as a safeguard it will be shared using Accellion which is a Secure File Transfer Appliance that provides a secure way to send large files to recipients both inside and outside the study setting.

\section{Data monitoring}


The Data and Safety Monitoring Board (DSMB) will comprise of the Principal Investigators. The DSMB will be responsible for study oversight, including monitoring safety, ensuring that the study is conducted according to the protocol and ensuring data integrity.

An internal committee to monitor data safety will be formed. The progress of enrollment and follow-up will be monitored monthly and needed reports submitted to the internal committee on data safety. The DSMB will meet a minimum of every 12 months and will in general align with the annual IRB continuation renewal. The DSMB will submit report to the UMB and Nigerian IRB at the conclusion of each meeting.

\section{Reporting unanticipated adverse effects}

Unanticipated problems that are serious adverse events will be reported to the UMB and Nigerian IRBs and to NICHD within 1 week of the investigator becoming aware of the event.

Any other unanticipated problem will be reported to the UMB and Nigerian IRB and to NICHD within 2 weeks of the investigator becoming aware of the problem.

All unanticipated problems will be reported to appropriate institutional officials (as required by an institution's written reporting procedures), the supporting agency head (or designee), and OHRP within one month of the IRB's receipt of the report of the problem from the investigator.

\section{Study staff recruitment and training}

Each site has identified an adult ART physician, a pediatric ART physician, a laboratory scientist, and a nurse familiar with the adolescents and involved in the management of their HIV care in the clinics. These will form the core site study staff. In addition, the site study staff will also recruit peer adolescents for the PTA and El intervention arms previously described above.

Trainings will include:

- A central training to familiarize site study staff and laboratory personnel on the study protocol and data collection tools, recruitment and enrolment of study participants as well as PTAs and Els, and to solicit feedback

- Another central level training for PTAs and Els on the study protocol, ethical considerations, standard operating procedure, specific duties per intervention arm, and data collection and management.

- Refresher trainings will be conducted annually.

\section{Discussion}

The ADAPT study will be testing a patient-centered approach to inform the development of an innovative study intervention. We hypothesize that patient engagement in the design of the study, follow up and in the definition of the outcomes, will result in an approach that will be sustained beyond the limits of the study. The generalizability of our findings is assured by implementing research intervention within existing systems and HIV care implementation groups across Nigeria.

\section{Strengths}


The multi-PI team for this study includes strong and complementary expertise in pediatric, adolescent and adult HIV care, epidemiology, and social science, with a documented presence and successfully conducting of research in Nigeria. The leveraging on the PEPFAR program environment in Nigeria enables broad study in implementation science. The long-standing collaboration with the Government of Nigeria through the Federal Ministry of Health, the National Action Committee on AIDS (NACA), and the National AIDS/STDs Control Program (NASCAP) will be important within the context of dissemination of findings and ensuring findings go to impact. The ADAPT study team has strong support for conducting this study from the University of Maryland School of Medicine faculty. The successful implementation, research and laboratory operations of our affiliated implementing partner, IHVN, will certainly guarantee smooth processes.

\section{Potential limitations}

We recognize a few potential limitations to this study. There are no research assistants to be directly responsible for data collection and transmission to the central office from the six study sites. To mitigate this, we plan to train the Nurse Coordinators at the sites on data collection and management and carry out bi-weekly oversight visits to the sites. Also, we made sure we recruited site staff who are very knowledgeable in the management of HIV care. Another potential limitation is that ALHIV for our study are mostly those perinatally infected, and as such this might diminish the generalizability of our findings to those who acquired the infection behaviorally.

\section{Potential Implications for Practice and Research}

One key implication for practice is that the adolescent patient-centered care is a successful intervention that is critical to the processes of retention in care and treatment in this vulnerable population. The patient's perception of patientprovider communication type of service provision is a potential area for research.

\section{Trial status}

This trial was registered with ClinicalTrials.gov (NCT02872805) on 12 May 2017. The formative phase of the study was completed on 06 April, 2018. We have received all IRB approvals needed to initiate Phase II of the study.

\section{Declarations}

\section{Acknowledgements}

The ADAPT team thanks the Nigerian Federal Ministry of Health and the Pediatric Association of Nigeria, and the Nigerian Ministry of Women Affairs for their support of this study. The authors acknowledge and appreciate the study coordinators, investigators and staff IHVN and the facilities (AKTH, ADH, UATH, FMC Keffi, DASH and GH Katsina) for their invaluable contributions to the study design and site assessments and selection.

\section{Funding}

The ADAPT trial is supported by the Eunice Kennedy Shriver National Institute of Child Health and Human Development of the National Institutes of Health under award number 1R01HD089866 to VT, MC and EE. The content is solely the responsibility of the authors and does not necessarily represent the official views of the National Institutes of Health. The funding agencies played no role in the study conception or design and will not play a role in data collection, data analysis, data interpretation, or writing of the report.

\section{Availability of data and materials}

Page 12/19 
Not applicable

\section{Authors' contributions}

VT and MC conceived the study and the design, contributed to data collection and manuscript draft, critically reviewed the manuscript for intellectual content and approved the final draft. EE contributed to the study and the design, contributed to data collection and manuscript draft, critically reviewed the manuscript for intellectual content and approved the final draft. $\mathrm{CE}, \mathrm{Cl}, \mathrm{AS}$ and $\mathrm{JJ}$ contributed to the design and data collection, critically reviewed the manuscript for intellectual content, and approved the final draft. $\mathrm{HO}$ contributed to the randomization process and statistical estimations, and approved the final draft. Site investigators $(\mathrm{FH}, \mathrm{BI}, \mathrm{ME}, \mathrm{EA}, \mathrm{AO}, \mathrm{HO})$ critically reviewed the manuscript for intellectual content and approved the final draft. All authors read and approved the final manuscript.

\section{Ethics approvals and consent to participate}

The ADAPT trial has been approved by the Institutional Review Board (IRB) of the University of Maryland (\#HP00070099), as well as the Nigerian National Health Research Ethics Committee (NHREC/01/01/2007-07/04/2017). Written informed consent will be obtained from parents/guardians and eligible emancipated minor ALHIV, and assent will be obtained from all eligible minor ALHIV. All IRBs will be notified of any modifications to the protocol.

\section{Competing interests}

The authors declare that they have no competing interests.

\section{Consent for publication}

Not applicable

Affiliations.

\section{Author details}

1Prevention, Care and Treatment Department, and International Research Center of Excellence, Institute of Human Virology Nigeria, Abuja, Nigeria. 2Division of Epidemiology and Prevention, Institute of Human Virology, University of Maryland School of Medicine, Baltimore, MD, USA. 3Pediatric AIDS Program, University of Maryland School of Medicine, Baltimore, MD, USA.

4Dalhatu Araf Specialist Hospital, Lafia, Nassarawa, Nigeria. 5Asokoro District Hospital, Abuja, Nigeria. 6General Hospital, Katsina, Nigeria. 7Federal Medical Center, Keffi, Nassarawa, Nigeria. 8Aminu Kano Teaching Hospital, Kano, Nigeria. 9University of Abuja Teaching Hospital, Abuja, Nigeria.

\section{References}

1. Adolescents and Young People UNICEF Data. https://data.unicef.org/topic/hivaids/adolescents-young-people/) 2017

2. Ruria EC, Masaba R, Kose J, Woelk G, Mwangi E, Matu L, Ng'eno H, Bikeri B, Rakhmanina N. Optimizing linkage to care and initiation and retention on treatment of adolescents with newly diagnosed HIV infection. AIDS. 2017 Jul 1;31 Suppl 3:S253-S260. doi:10.1097/QAD.0000000000001538.

3. NACA (2015) 'Nigeria GARPR 2015' (pdf) 
4. Idele P, Gillespie A, Porth T, Suzuki C, Mahy M, Kasedde S, et al. Epidemiology of HIV and AIDS among adolescents: current status, inequities, and data gaps. J Acquir Immune Defic Syndr. 2014;66 (Suppl 2):S144-49. [PubMed].

5. Nigeria Federal Ministry of Health (2013) 'National HIV \& AIDS and Reproductive Health Survey 2012, (NARHS Plus II) (pdf).

6. Kapogiannis BG, Legins KE, Chandan U, Lee S. Evidence-Based Programming for Adolescent HIV Prevention and Care: Operational Research to Inform Best Practices. JAIDS Journal of Acquired Immune Deficiency Syndromes. 2014;66:S228-S235. [PubMed]

7. Philbin, Morgan \& E. Tanner, Amanda \& L. DuVal, Anna \& M. Ellen, Jonathan \& Xu, Jiahong \& Kapogiannis, Bill \& Bethel, Jim \& Dennis Fortenberry, J. (2013). Linkage to Care and Engagement in Care for Newly Diagnosed HIVPositive Youth Within Fifteen Adolescent Medicine Clinics in the United States. AIDS and Behavior. 18. 10.1007/s10461-013-0650-6.

8. Philbin MM et al. HIV testing, care referral, and linkage to care intervals affect time to engagement in care for newly diagnosed HIV-infected adolescents in 15 Adolescent Medicine Clinics in the United States. J Acquir Immune Defic Syndr, 72: 222-29, 2016.

9. Inwani I, Chhun N, Agot K, Cleland CM, Buttolph J, Thirumurthy H, Kurth AE High-Yield HIV Testing, Facilitated Linkage to Care, and Prevention for Female Youth in Kenya (GIRLS Study): Implementation Science Protocol for a Priority Population JMIR Res Protoc 2017;6(12):e179 DOI: 10.2196/resprot.8200

10. Health for the world's adolescents: a second chance in the second decade Geneva, Switzerland: World Health Organization; 2014 [cited 2017 January 6]. Available from: http://apps.who.int/adolescent/second-decade/files/ 1612_MNCAH_HWA_Executive_Summary.pdf

11. Herek GM, Capitanio JP, Widaman KF. HIV-related stigma and knowledge in the United States: Prevalence and trends, 1991-1999. Am J Public Health 2002;92:371-377 [PMC free article][PubMed]

12. Gilliam PP, Ellen JM, Leonard L, Kinsman S, Jevitt CM, Straub DM. Transition of Adolescents With HIV to Adult Care: Characteristics and Current Practices of the Adolescent Trials Network for HIV/AIDS Interventions. Journal of the Association of Nurses in AIDS Care. 2011;22(4):283-294.https://doi.org/10.1016/j.jana.2010.04.003. [PMC free article] [PubMed]

13. Valenzuela JM, Buchanan CL, Radcliffe J, Ambrose C, Hawkins LA, Tanney M, Rudy BJ. Transition to Adult Services among Behaviorally Infected Adolescents with HIV-A Qualitative Study. Journal of Pediatric Psychology. 2011;36(2):134-140. https://doi.org/10.1093/jpepsy/jsp051. [PubMed]

14. Kakkar F, Van Der Linden D, Valois S, Maurice F, Onnorouille M, Lapointe N, et al. Health outcomes and the transition experience of HIV-infected adolescents after transfer to adult care in Quebec, Canada. BMC Pediatr. 2016;16:109.

15. Dowshen N, D'Angelo L. Health Care Transition for Youth Living With HIV/AIDS. PEDIATRICS. 2011;128(4):762771. https://doi.org/10.1542/peds.2011-0068. [PubMed]

16. Tanner AE, Philbin MM, Duval A, Ellen J, Kapogiannis B, Fortenberry JD The Adolescent Trials Network for HIV/AIDS Interventions. "Youth friendly" clinics: Considerations for linking and engaging HIV-infected adolescents into care. AIDS Care. 2014;26(2):199-205.https://doi.org/10.1080/09540121.2013.808800. [PMC free article] [PubMed] 
17. Fair CD, Sullivan K, Dizney R, Stackpole A. "It's Like Losing a Part of My Family": Transition Expectations of Adolescents Living with Perinatally Acquired HIV and Their Guardians. AIDS Patient Care and STDs. 2012120611131552000. https://doi.org/10.1089/apc.2012.0041. [PubMed]

18. NACA (2015) 'Nigeria GARPR 2015’ (pdf)

19. Johnson LF, Davies MA, Moultrie H, Sherman GG, Bland RM, Rehle TM, et al. The effect of early initiation of antiretroviral treatment in infants on pediatric AIDS mortality in South Africa: a model-based analysis. Pediatr Infect Dis J. 2012;31(5):474-480. [PubMed]

20.

http://www.ich.org/fileadmin/Public_Web_Site/ICH_Products/Guidelines/Efficacy/E6_R1/Step4/E6_R1_Guideline.pdf 21. Federal Ministry of Health Nigeria, 2016. National Guidelines for HIV Prevention, Treatment and Care. Available at: https://aidsfree.usaid.gov/sites/ default/files/ng_national_guidelines_hiv.pdf. Accessed May 2017

22. Hibbard JH, Mahoney ER, Stockard J , Tusler M . Development and testing of a short form of the patient activation measure. Health Serv Res . 2005 ; 40 ( 6 Pt 1 ): 1918 - 30 .

23. Alderfer M.A., Marsac M.L. (2013) Pediatric Quality of Life Inventory (PedsQL). In: Gellman M.D., Turner J.R. (eds) Encyclopedia of Behavioral Medicine. Springer, New York, NY

24. Harold D. Green, Jr., Matthew A. Hoover, Glenn J. Wagner, Gery W. Ryan, and Eric Ssegujja. Measuring Agreement between Egos and Alters: Understanding Informant Accuracy in Personal Network Studies Field Methods. Vol 26, Issue 2, pp. $126-140$

25. Eldridge S Kerry S A Practical Guide to Cluster Randomised Trials in Health Services Research Chichester, UK Wiley, 2012

26. Donner A, Klar N, Design and Analysis of Cluster Randomization Trials in Health Research Chichester, UK, Wiley, 2000.

27. Schulz KF, Altman DG, Moher D; CONSORT Group. CONSORT 2010 statement: updated guidelines for reporting parallel group randomised trials. BMJ. 2010 Mar23;340:c332

28. Institute of Medicine. "Crossing the Quality Chasm: A New Health System for the 21st Century"

29. Nicolaie M. A. and van Houwelingen, H. C. and de Witte, T. M. and Putter, H. (2012). Dynamic prediction in competing risks by landmarking. Stat Med, in press.

30. C. Hawkins, G. Chalamilla, J. Okuma, D. Spiegelman, E. Hertzmark, A. Aris, et al.Sex differences in antiretroviral treatment outcomes among HIV-infected adults in an urban Tanzanian setting AIDS, 25 (2011), pp. 1189$1197,10.1097 /$ QAD.0b013e3283471deb

\section{Figures}




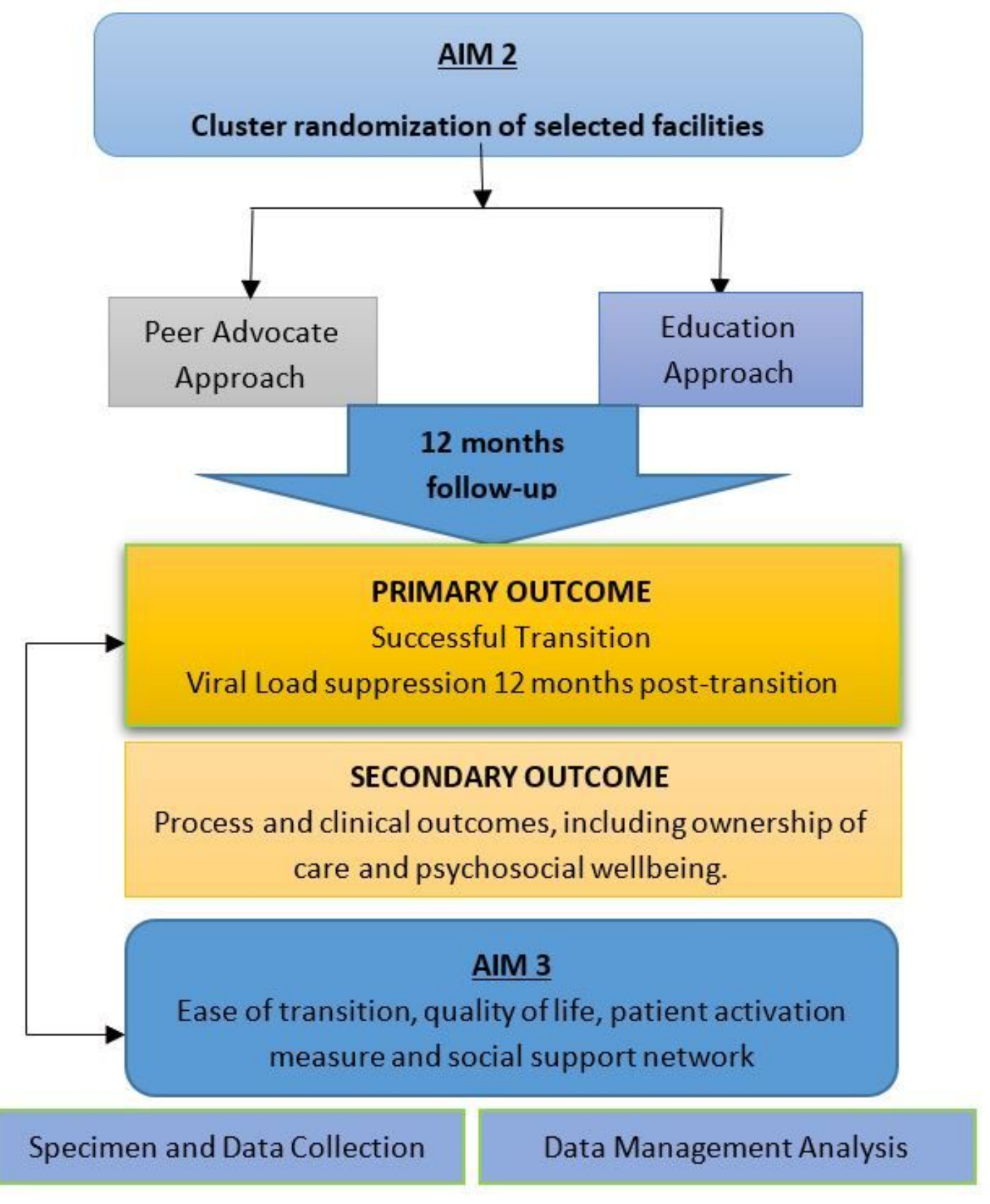

Figure 1

ADAPT Intervention Design and Framework 


\section{Site Recruitment}

Recruiting from 6 PEPFAR-supported facilities

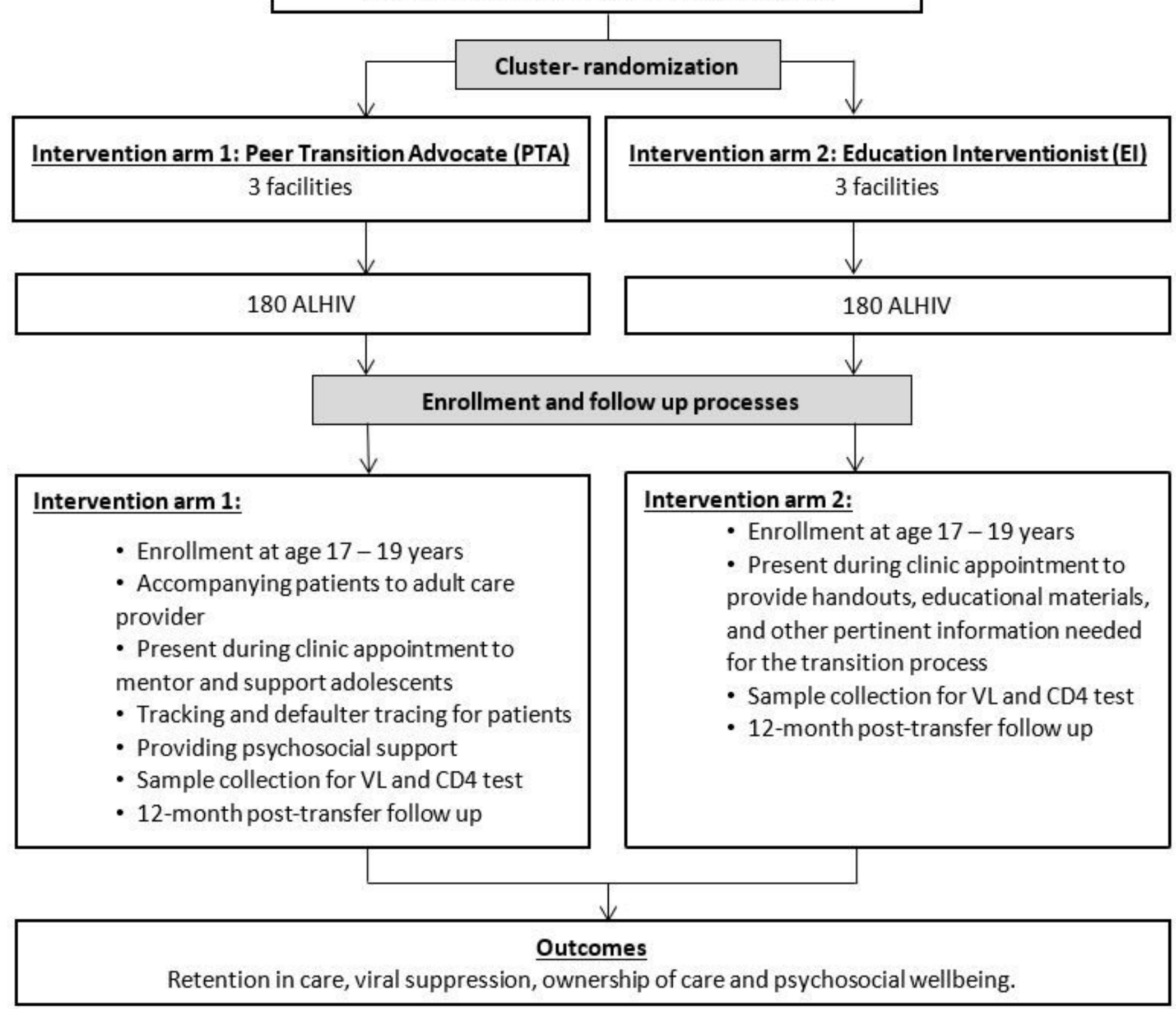

Figure 2

Enrollment and Follow-up Processes 


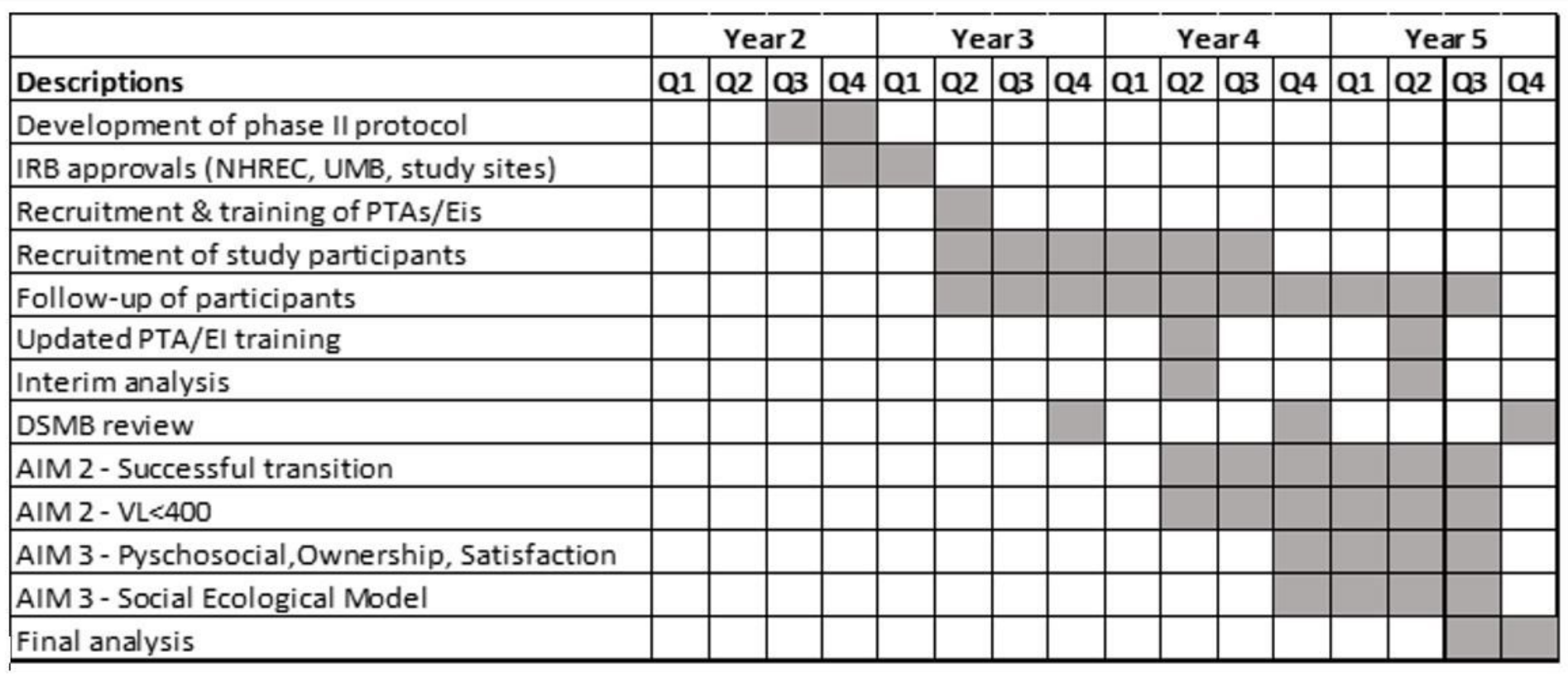

Figure 3

Proposed intervention timeline

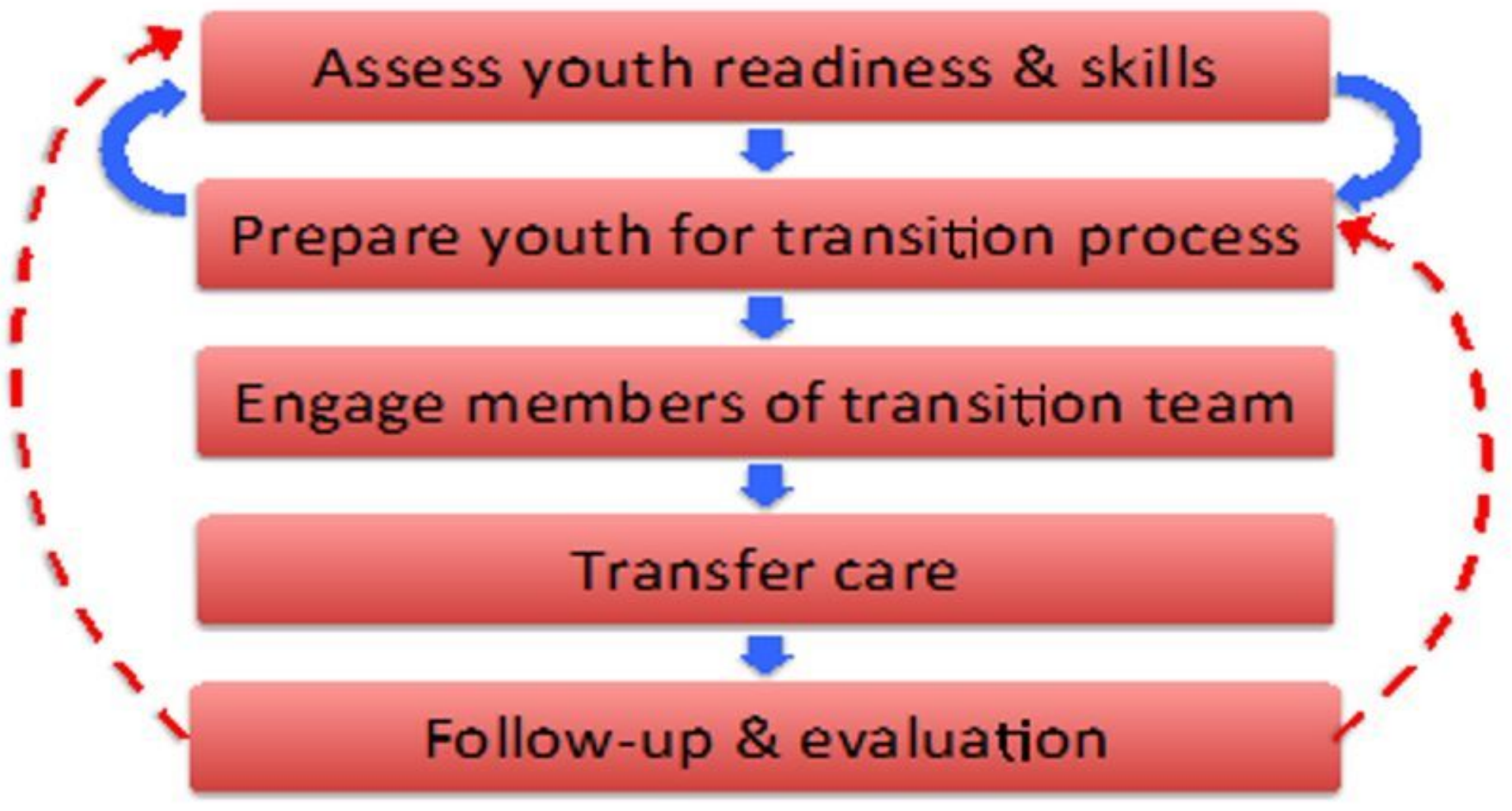

Figure 4

Basic Steps in Transition Pathway

\section{Supplementary Files}


This is a list of supplementary files associated with this preprint. Click to download.

- ADAPTStudySPIRITChecklist12242018.doc 